\title{
Students, Medicines and Performance Consumption: The Online as a Source of Information and Sharing ${ }^{\dagger}$
}

\author{
Catarina Egreja *(D) and Noémia Lopes (D) \\ Instituto Universitário Egas Moniz, 2829-511 Almada, Portugal; nlopes@egasmoniz.edu.pt \\ * Correspondence: cegreja@egasmoniz.edu.pt \\ t Presented at the 5th International Congress of CiiEM-Reducing Inequalities in Health and Society, Online, \\ 16-18 June 2021.
}

check for

updates

Citation: Egreja, C.; Lopes, N. Students, Medicines and Performance Consumption: The Online as a Source of Information and Sharing. Med. Sci. Forum 2021, 5, 10. https://doi.org/ 10.3390/msf2021005010

Academic Editors: Helena Barroso and Cidália Castro

Published: 20 July 2021

Publisher's Note: MDPI stays neutral with regard to jurisdictional claims in published maps and institutional affiliations.

Copyright: (c) 2021 by the authors. Licensee MDPI, Basel, Switzerland. This article is an open access article distributed under the terms and conditions of the Creative Commons Attribution (CC BY) license (https:// creativecommons.org/licenses/by/ $4.0 /)$.

\begin{abstract}
We aim to reflect on the online as a space to be taken into account in the analysis of sources of information about medicines, as a means of transmitting knowledge and practices among students, by presenting results of a content analysis based on material collected from blogs and internet forums. We conclude that sharing experiences is central to the validation of and willingness to use these resources. Considering the consequences that may arise from widespread use of the online as a source of information for therapeutic or performance purposes is important.
\end{abstract}

Keywords: medicines and supplements; online information sources; online sharing; performance enhancement in students

\section{Introduction}

Previous research concludes that the internet serves as a space for users to gain some expertise about medicines, being able to exchange ideas and ask questions. Users seek, in forums, to collect experiences from other users, and peer support in the search for a shared social identity; the higher the risk associated with health, the more the interest grows [1]. Online forums are valued because of the anonymity-being able to communicate without fear or worry and choosing how much information to share [2]. The growth of the internet and the increased use of forums means that users now have access to a wider range of views and opinions on their chosen topic $[3,4]$.

The present exploratory approach reflects on the online as a space that should be taken into account in the analysis of sources of information on medicines, as a means of transmission of knowledge and practices among users, considering its potential and limits, by presenting the results of a content analysis based on material collected from blogs and internet forums. Objectively, the focus is on online information exchanges among high school and university students, on the consumption of medicines and natural products, with the purpose of improving the capacity to concentrate and memorize during exams. Although this is not a new research theme, its pertinence and topicality merit an opportunity to deepen the analysis.

\section{Materials and Methods}

The adopted methodology was qualitative content analysis. In order to find online information exchange practices on the consumption of medicines and natural products, the research process began by entering keywords into a search engine (Google). We did not intend to explore sites of pharmacies or companies linked to this area, nor to find sources of information that came from experts (doctors, pharmacists, etc.), but rather interactions between lay people, in this case, students. These were found in different forums and blogs, mostly of student associations, where participants sometimes asked questions about issues related to these consumptions. 
Next, we collected all the posts and respective comments of interest, which were categorized and examined relying on an analytic grid with the following structure, using the Maxqda software: (1) Sharing experiences-symptoms; therapeutic path (consumption, dosages, reactions...); request for help/clarification of doubts; (2) Sharing expert knowledge-diagnoses; recommended therapy; (3) Sharing lay knowledge-diagnoses; effects of medicines/supplements; consumption advices; advices of consultation with health professionals/others; other advices.

On the topic of consumption of performance-enhancing medicines and supplements among students, 93 conversation excerpts ranging from March 2015 to October 2020 were analysed.

\section{Results and Discussion}

The results show that one of the main reasons for students to use blogs and forums as a source of information is the possibility to share experiences (31.2\%). One of the most frequent aspects concerns requests for help, mainly in order to obtain information and clarify doubts. Our analysis also indicates that students seek to know the opinion of their peers regarding the taking of supplements or medication to help them study, in order to obtain some kind of validation. This generates, in turn, a sharing of their own or other people's experiences, through the account of therapeutic paths (consumption, dosages, and reactions). However, the dimension of analysis with the highest proportion of content $(63.4 \%)$ refers to the sharing of lay knowledge. In response to doubts or requests for information, the other participants in these virtual communities take the opportunity to offer their diagnoses, tell the effects of the medicines or supplements they think they know, or recommend ways of consumption. Finally, the dimension with the least expression is the sharing of expert knowledge (5.4\%). Although this is not a common situation, some people copy information from package leaflets, a reliable site or scientific studies to present the effects and purposes of medicines in an expert way, not based on their personal convictions.

In conclusion, these means of access to information clearly reveal that the sharing of experiences is central to the validation and willingness to resort to such consumption. It is important to reflect on the consequences that may arise from the generalisation of these means as sources of information for therapeutic or performance purposes-not only in the case of students, but more broadly, by the population in general.

The study has limitations, such as the impossibility to know for sure if the online participants are, indeed, lay students, or the inability to characterize the sample. However, we believe that the aspects pointed out here justify a deepening of this line of research, also to strengthen current studies on the concept of medication literacy.

Institutional Review Board Statement: The study was conducted according to the guidelines of the Declaration of Helsinki, and approved by the Ethics Committee of Instituto Universitário Egas Moniz (protocol code CE 857/20.02.2020).

Informed Consent Statement: Not applicable.

Data Availability Statement: Not applicable.

Acknowledgments: This work is part of project ConPerLit (PTDC/SOC/30734/2017), funded by the Portuguese Foundation for Science and Technology.

Conflicts of Interest: The authors declare no conflict of interest.

\section{References}

1. Dresen, A.; Kläber, M.; Dietz, P. Use of performance-enhancing drugs and the Internet. Criminological reflections on a culture of communication in sport. Sportwissenschaft 2014, 44, 153-159. [CrossRef]

2. Fage-Butler, A.M.; Jensen, M.N. The Interpersonal Dimension of Online Patient Forums: How Patients Manage Informational and Relational Aspects in Response to Posted Questions. Hermes J. Lang. Commun. Bus. 2013, 51, 21-38. [CrossRef] 
3. Tighe, B.; Dunn, M.; McKay, F.H.; Piatkowski, T. Information sought, information shared: Exploring performance and image enhancing drug user-facilitated harm reduction information in online forums. Harm Reduct. J. 2017, 14, 1-9. [CrossRef] [PubMed]

4. Rusu, I.A. Exchanging health advice in a virtual community: A story of tribalization. J. Comp. Res. Anthropol. Sociol. 2016, 2, 57-69. 